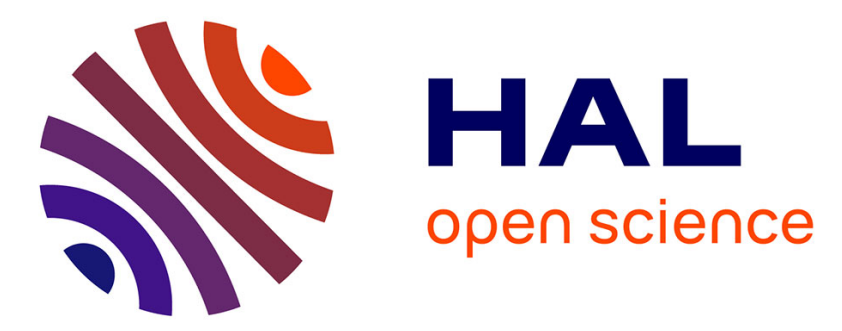

\title{
Ganglioside GM3 and Its Role in Cancer
}

Changping Zheng, Marco Terreni, Matthieu Sollogoub, Yongmin Zhang

\section{To cite this version:}

Changping Zheng, Marco Terreni, Matthieu Sollogoub, Yongmin Zhang. Ganglioside GM3 and Its Role in Cancer. Current Medicinal Chemistry, 2019, 26 (16), pp.2933-2947. 10.2174/0929867325666180129100619 . hal-02889373

\section{HAL Id: hal-02889373 https://hal.science/hal-02889373}

Submitted on 22 Oct 2020

HAL is a multi-disciplinary open access archive for the deposit and dissemination of scientific research documents, whether they are published or not. The documents may come from teaching and research institutions in France or abroad, or from public or private research centers.
L'archive ouverte pluridisciplinaire HAL, est destinée au dépôt et à la diffusion de documents scientifiques de niveau recherche, publiés ou non, émanant des établissements d'enseignement et de recherche français ou étrangers, des laboratoires publics ou privés. 


\section{Ganglioside GM3 and Its Role in Cancer}

Changping Zheng ${ }^{\text {a }}$, Marco Terreni ${ }^{\mathrm{c}}$, Matthieu Sollogoub ${ }^{\mathrm{a}}$, Yongmin Zhang ${ }^{\mathrm{a}, \mathrm{b},{ }^{*}}$ ${ }^{a}$ Sorbonne Universités, UPMC Univ Paris 06, CNRS, Institut Parisien de Chimie Moléculaire (UMR 8232), 4 Place Jussieu, 75005 Paris, France; ${ }^{b}$ Institute for Interdisciplinary Research, Jianghan University, Wuhan Economic and Technological Development Zone, 430056 Wuhan, China; ${ }^{c}$ Drug Sciences Department, University of Pavia, Via le Taramelli 12, 27100 Pavia, Italy.

* Corresponding author

Sorbonne Universités, UPMC Univ Paris 06, CNRS, UMR 8232, IPCM, 4 place Jussieu 75005 Paris, France. E-mail: yongmin.zhang@upmc.fr 
Abstract: Ganglioside GM3 is strongly related with human tumors, such as lung, brain cancers and melanomas, and more and more evidences have revealed that GM3 possesses powerful effects on cancer development and progression. GM3 is over expressed on several types of cancers, and can be as a tumor-associated carbohydrate antigen, used for immunotherapy of cancers. GM3 can also inhibit tumor cells growth by anti-angiogenesis or motility and so on. Especially, GM3 has effects on the EGFR tyrosine kinase signaling, uPAR-related signaling and glycolipid-enriched microdomains, which are essential for cancer signaling conduction. It is obvious that GM3 will be a promising target for cancer treatment.

Keywords: GM3, antitumor, immunotherapy, EGFR, uPAR-related signaling, anti-angiogenesis, cell motility, GM3-enriched microdomains. 


\section{INTRODUCTION}

Glycosphinolipids (GSLs) are ubiquitous components on animal cell membranes, and are exposed on the outer surface. The studies have demonstrated that they play a role in cell proliferation, adhesion, motility and differentiation [1]. Gangliosides, a family of GSLs, have been shown to be involved in the development and progression of cancers [2,3]. GM3, the structurally simple monosialoganglioside, contains a single terminal sialic acid, lactose and ceramide (Figure 1).

Figure 1

The obvious influence of GM3 on the proliferation, invasion and metastasis of tumor cells has been observed [4-6]. GM3 is over expressed on some cancers, and it is well-known as a tumor-associated carbohydrate antigen on several types of tumors, which can be used for developing cancer vaccines. Furthermore GM3 can modulate the receptors or signaling pathways related to cancers, including epidermal growth factor receptor (EGFR) and uPAR-related signaling. In addition, GM3 can also inhibit tumor cells growth by anti-angiogenesis or motility. In particular, GM3 can modulate functions of other molecules co-localized in membrane microdomains. Until now, many types of cancers associated with GM3 have been studied (Table 1), and the clear effects of GM3 on tumor cells growth have been observed. Besides, some exogenous GM3 analogues can also influence cancer cells growth. For example, Qu. et al. accomplished the synthesis of GM3 analogues 1 and 2 (Figure 2) [7], and the data showed that in a cytotoxicity assay, the analogues are actively against the human HCT116 (human colon carcinoma cells) and K562 (chronic myelogenous leukemia) cancer cells.

Table 1

Based on these observations, the study of GM3 with antitumor properties appears to be a logical matter of research, and this review describes the current research achievements on GM3 and carcinomas.

Figure 2

\section{GANGLIOSIDE GM3 EXPRESSION ON TUMORS}

Many studies have shown that GM3 expression is strongly related to human cancers, and change of GM3 expression is associated with the growth and invasion of tumor cells [15,16]. NEU3 is a plasma membrane-associated sialidase, and its selective substrates are ganglioside GM3 and GD1a $[17,18]$. Studies revealed that its expression is up-regulated in renal cell carcinoma, colonic cancer and 
melanoma $[19,20]$. Further studies indicated that the effect of sialidase on ganglioside expression appears to play an important role in the cancer progression through promoting invasive potential or inhibiting apoptosis [21]. Although sialidase over expression and then decreased GM3 content have been detected in some tumors, low levels of sialidase and increased GM3 content have been found in highly metastatic variants of colon adenocarcinomas, and sialidase over expression can inhibit cell growth and metastasis [22].

GM3 expression on the melanomas is the most widely studied. It has been reported that GM3 is expressed with a much higher percentage in the primary and metastatic melanomas than GD2, and that GM3 and GD3 expressions are strongly related to each other in primary and metastatic melanomas. GM3 can not be found in normal skin or on skin neighboring primary melanoma, which is in contrast with GD3. GM3 expression starts at an early stage of melanocyte differentiation, which is associated with allowing entry into the dermis of the skin $[23,24]$. GM3 is a major ganglioside in many mammalian cells and more exposed in the strongly metastatic line than the weakly metastatic line on the melanomas. Furthermore, mutant clones that have lost the GM3 expression show lower metastatic potential [25]. It is clear that GM3 expression on the tumor cell surface is an important factor in determining the metastatic phenotype in cancer cells [26].

Cancer cells growth can be stimulated under the condition of GM3 depletion, whereas GM3 increase can lead to suppression. In the murine MBT-2 bladder tumors, the exogenous GM3 can inhibit tumor growth and invasion [12,14]. A large quantity of GM3 is accumulated in nonmuscle-invasive bladder cancer, but a small quantity of GM3 is accumulated in muscle-invasive bladder tumor. The enhanced GM3 expression caused by brefeldin A can also lead to inhibition of bladder cancer cells growth. Additionally, in murine bladder cancer, over expression of GM3 by GM3 synthase resulted in apoptosis and reduced the malignant potential [12]. It is obvious that the exogenous or endogenous GM3 has a significant antitumor effect on bladder cancer. Next, human gliomas have also been studied. The results showed that the sparse and confluent C6 glioma cells differ in GM3 expression and content, which increase with cell density. Furthermore, in endothelin-1 (ET-1)-induced phosphoinositide hydrolysis, GM3 content is much higher in the sparse cells than in confluent ones [27]. Studies also suggest that rapidly proliferating tumor cells are more sensitive to GM3 treatment than normal neural cells, and GM3 treatment may have therapeutic potential for high-grade human gliomas [9].

N-glycolyl-GM3 contains N-glycolylneuraminic acid instead of N-acetylneuraminic acid, and it 
is widely expressed in most mammals, but not in normal human cells [28]. However, it has been detected in many human cancers, such as colon carcinomas and breast cancers, and it can be considered as one of potential target molecules in cancer therapy [29,30]. The detailed mechanisms of its expression and functions in human cancers are still to be explored.

\section{GANGLIOSIDE GM3 BASED CANCER VACCINES}

A study demonstrated that the silencing of the cmah gene in L1210 mouse lymphocytic leukemia B cells caused obvious change about GM3 expression resulting in a reduction of tumorigenicity [31]. It is known that GM3 is a tumor-associated carbohydrate antigen on several types of cancer. Targeting ganglioside GM3 antigen has been a matter of research to study because their possibility of leading to autoimmune responses. Hanganutziu-Deicher (HD) antibodies in the healthy human serum recognize glycoconjugates exhibiting NeuGc. Auto-antibodies against tumor-associated antigens can arise and be detected early before symptoms occur, which has a potential application for early diagnosis [32,33]. A study also reported that healthy humans exhibited low levels of anti-NeuGc GM3 antibodies. However, these antibodies were absent in non-small-cell lung cancer (NSCLC) patients [34,35]. In fact, antibodies reactive to NeuGc-containing glycolipids have been found to be induced in patients after repeated transfusions with sera from other species in melanoma patients [36]. Melanoma cells over-express GM3, and natural anti-NeuGc antibodies are increased in melanoma patients [37]. Especially, a higher level of anti-NeuGc antibodies was detected in patients who were free of disease within more than 5 years than 2 years after surgery [38].

Cancer immunotherapy is a highly promising approach to cancer treatment, and has been gaining more and more attention recently. Targeting tumor-associated GM3 has been already investigated to develop vaccines. It has been confirmed that derivatized GM3 antigens are generally more immunogenic than GM3 itself [39]. While GM3 induces mainly IgM responses, some derivatized GM3 antigens induce both $\operatorname{IgM}$ and $\operatorname{IgG}$ responses. Based on the viewpoint about cancer therapy, $\operatorname{IgG}$ responses are more pregnant due to their desirable properties such as immunological memory and affinity.

A series of structurally modified GM3 antigens and their protein conjugates were designed and synthesized, then these derivatized antigens were studied to explore the impact on immunogenicity. The results on mice showed that suitable modifications on the GM3 can improve the immunogenicity. 
Especially, N-modified derivatives of GM3 and their protein conjugates were prepared. GM3NPhAc proved to own better immunogenicity among the GM3 N-derivatives investigated, and it is the least cross-reactive with the natural GM3 [40]. GM3NPhAc is an excellent candidate as an anti-tumor vaccine. Meanwhile, the combination of a GM3NPhAc-based vaccine with the glycoengineering of cancer cells by $\mathrm{N}$-phenylacetylmannosamine provides great promises as an effective cancer immunotherapy [40].

Among N-glycolylated gangliosides, the NGcGM3 has been particularly studied. This molecule has been evaluated as a target for cancer immunotherapy because its preferential expression on human malignant cells and its possible involvement in cancer progression based on immunosuppressive effects [41,42]. The NGcGM3 has been described in human neoplasms, including breast carcinoma and melanoma, but is usually not detected in normal human cells [43,44]. So it makes NGcGM3 as an interesting target for immunotherapy. Now, NGcGM3/VSSP, an NGcGM3 ganglioside conjugated with very small proteoliposomes has been developed. In the clinical trials, the NGcGM3/VSSP vaccine showed efficacy and was well-tolerated by patients [45-47]. Similarly to the NGcGM3/VSSP vaccine, the antibodies induced by racotumomab treatment interacted with GM3 murine tumor cell line and lung carcinoma tissue sections [43]

\section{GANGLIOSIDE GM3 IN EGFR SIGNALING PATHWAY}

Epidermal growth factor (EGF) is an important cytokine regulating various cell processes, including cell proliferation, differentiation, apoptosis and oncogenesis. The EGF can bind to EGFR resulting in dimerisation, autophosphorylation, and subsequently downstream signaling (Figure 3). Over activation of epidermal growth factor receptor (EGFR) is well known to be associated with cancer development and progression. EGFR tyrosine kinase is regarded as an important target to search the effective pharmacological compounds that can inhibit the growth of cancer cells, since the majority of human cancers are derived from epidermal tissue. Enhanced tumor cells growth and invasion are controlled by enhanced EGFR tyrosine kinase, and thus, EGFR is a promising target for cancer therapy [48].

\section{Figure 3}

Many studies have demonstrated that GM3 has an inhibitory effect on EGFR. In human epidermoid carcinoma A431 cells, exogenous addition of GM3 inhibited EGF-induced 
autophosphorylation of EGFR [8]. GM3 has been known to inhibit EGFR tyrosine kinase since 1986 [49,50]. Further research results showed that GM3 inhibits EGFR tyrosine kinase through binding to N-linked glycan having multivalent GlcNAc termini on EGFR [51]. It is important to note that GM3 susceptibility for EGFR is strongly dependent on the presence of N-glycosylation because tunicamycin treatment abolishes GM3 susceptibility [52]. This is a kind of carbohydrate to carbohydrate interaction within the same membrane plane and presumably within the same glyco synaptic microdomain.

Studies also indicate that receptor dimer formation is not strongly blocked by GM3, but tyrosine phosphorylation on both monomeric and dimeric EGFR is inhibited by GM3, and the dose-dependent inhibitory effect on tyrosine phosphorylation in both monomeric and dimeric EGFR was observed [53]. Besides, other studies further determined the effect of GM3 on the Tyr-1173 and Tyr-1086 residues phosphorylation of EGFR. The results indicated that GM3 suppressed the phosphorylation of Tyr-1173 residue, and enhanced the phosphorylation of Tyr-1086 residue. Both Tyr-1173 and Tyr-1086 residues, which located at EGFR's C-terminal, are important EGFR autophosphorylation sites [54,55].

The importance of the sialosyl group of GM3 for its EGFR inhibitory effect was also studied. For A431 cells, they were transfected with a gene, which encodes for a soluble $\mathrm{Mr} 42,000$ sialidase. In comparison with control cells, although having similar level of EGF binding, the transfected cells could decrease sialic acid expression, promote cells growth, and enhance EGFR autophosphorylation [13]. Miyagi's group demonstrated that the enhanced expression of NEU3 leads to suppression of apoptosis and decreased NEU3 expression can inhibit Ras activation. Further, EGFR kinase inhibitor can block Ras activation by NEU3, and NEU3 over expression enhances EGFR autophosphorylation. Moreover, the involvement of NEU3 up regulation in cancer development can also be confirmed from increased EGFR phosphorylation detected in NEU3 transgenic mice [20,21,56,57].

Next, the order of binding intensity with some gangliosides is GM3 >> GM2, GD3, GM4 > GM1, GD1a, GD1b, GT1b, GD2, GQ1b > LacCer, consistent with the inhibitory effect of these gangliosides on EGFR activation [58]. GM3 exhibits the strongest effect on inhibiting EGFR among this family. Furthermore, GM3 derivatives were also tested on the EGFR activation. Among the gangliosides, de-N-acetyl-GM3 enhanced EGFR activity and EGF-induced cells growth, including A431, Swiss 3T3 and B16 cells. GM3 inhibited tyrosine phosphorylation, but de-N-acetyl-GM3 enhanced serine phosphorylation of EGFR [59]. We showed that mono-chloro-GM3 has a stronger inhibitory effect than GM3 on EGFR activation, and mono-chloro-GM3 inhibits activation of a mutant EGFR, $\triangle \mathrm{EGFR,}$ 
which is often detected in human glioblastoma cells [60].

The inhibitory effects of lipid mimetics of lyso-GM3 dimer on EGFR tyrosine kinase and various consequent phenotypic responses of A431 cells were also studied in detail [61]. The results showed that the lipid mimetic of lyso-GM3 dimer (Figure 4) and authentic lyso-GM3 dimer showed similar inhibitory effects. EGFR tyrosine kinase can not discriminate between the authentic structures and the lipid mimetic. Furthermore, a similar dose dependent inhibitory effect of the lipid mimetic of lyso-GM3 dimer on both monomeric and dimeric EGFR was also observed. Akt kinase can be activated by EGF, and its activity was inhibited by the lipid mimetic of lyso-GM3 dimer in A431 cells. It has been known that inhibition of the EGFR-dependent pathway can lead to perturbation of cell cycle progression and notably G1 arrest. The lipid mimetic of lyso-GM3 dimer inhibits EGFR tyrosine kinase, leading to inhibition of mitogenesis in A431 cells.

\section{Figure 4}

GM3 is the main ganglioside for Hepa1-6 cells, which can suppress the EGF-induced autophosphorylation of EGFR at tyr1173 and subsequently down regulated the phosphatidylinositide 3-kinases/Protein kinase B (PI-3K/Akt) signaling pathway [62]. However, GM3 can enhance the hepatocyte growth factor (HGF) induced autophosphorylation of HGFR at Tyr-1313 and Tyr-1365 and subsequently upregulates the PI-3K/Akt signaling pathway [63]. It shows that GM3 has an opposite effect on the EGF and HGF-induced Hepa1-6 cells motility and migration [64]. Indeed, GM3 can play a role on tumor progression through different ways, especially for EGFR activity. Not only GM3 can directly influence the EGFR activity by interaction with N-linked GlcNAc termini on the receptor, it also indirectly regulates EGFR activity through the interaction between EGFR and membrane proteins such as integrins and CD9 or influencing the intracellular Src kinase and protein tyrosine phosphatase

activity. It seems that the mechanism of GM3 modulating EGFR activity is rather complex. The co-expression of EGFR and NGcGM3 ganglioside in different human tumors has been reported [65]. NGcGM3 and EGFR can coordinately contribute to the tumor cell biology and combinations against these two targets can be a valid strategy for therapy.

\section{EFFECT OF GANGLIOSIDE GM3 ON UPAR-RELATED SIGNALING}


Urokinase plasminogen activator (uPA), a serine protease, has the capability to bind to its cell membrane receptor (UPAR), and thereby it initiates signal transduction events that lead to remodeling of extracellular matrices, cell migration, invasion, and proliferation in cooperation with integrins and other transmembrane partners [66]. A wide variety of human tumors, including melanomas, cutaneous carcinomas, and neoplasms of the breast, lung and brain, show increased expression or activation of uPA and UPAR. GM3 in the membrane rafts participates in the regulation of uPAR signaling, and plays a role in tumor cell growth.

The mechanism of GM3-triggered uPAR activation and cell proliferation was studied (Figure 5) [67]. uPAR associates with integrin $\alpha 5 \beta 1$ to cross-activate EGFR signaling in the presence of uPA [68]. uPA can be able to stimulate cell proliferation by either ERK-dependent or ERK-independent pathways. Cells show increased proliferation in the presence of uPA despite GM3 is increased or decreased. However, the underlying mechanism of increasing cell proliferation is different in the presence of increased GM3 and the absence of GM3. In contrast to (a) control cells, cells that (b) over express GM3 show activation of PI-3K and Protein kinase C PKC $\zeta$, increased p70S6 kinase activity, and cell proliferation, despite suppression of ERK signaling. Cells in which GM3 is (c) depleted show activation of ERK signaling, leading to increased cell proliferation that is independent of p70S6 kinase activation [69].

Figure 5

\section{EFFECT OF GANGLIOSIDE GM3 ON TUMOR ANGIOGENESIS}

The ratio of GM3 to a variety of gangliosides (GM1, GD3, GD1a, GT1b) can influence the angiogenic properties on many brain tumor types. The ratio of GM3 to the proangiogenic gangliosides GD3 and GD1a (GM3/GD3; GM3/GD1a) is lower in more metastatic and aggressive tumors than that in less metastatic tumors [70-72]. In mouse brain tumors or in most cultured human brain tumor cells, GM3 expression serves to regulate or to counteract the proangiogenic action of GD3 and other complex gangliosides [11]. Studies revealed that brain tumors with high GM3 expression are less angiogenic $[73,74]$. Other studies showed that removing GM3 expression gene in the ependymoblastoma tumor that contains the only ganglioside GM3, increased angiogenesis. And in the highly angiogenic CT-2A astrocytoma, decreased angiogenesis was observed when GM3 expression was upregulated (Figure 6) $[10]$. 


\section{Figure 6}

Next, it has been reported that uPAR localization in caveolar lipid-rafts (LRs) is indispensable for an efficient angiogenesis program in EPCs (endothelial progenitor cells) / ECFCs (endothelial colony-forming cells), commonly considered the main source of vasculogenesis in human cancers [75,76]. Further studies have found that uPAR is present on the ECFC surface in at least three compartments, one associated to GM1, another associated to GM3 and a third one associated to caveolar-LRs. Following GM1 exogenous addition, the GM3 compartment is depleted of uPAR which is recruited within caveolar-LRs thereby triggering angiogenesis-related transduction pathways that eventuate in enhanced ECFC invasion and capillary morphogenesis [77].

Further evidences indicated that GM3 can block the growth stimulation of vascular endothelium due to the reduced ability of the endothelial cell membrane to transfer growth signals present in the microenvironment into the endothelial cell itself [78]. Several neoplastic cells, especially melanomas, shed GM3 and other gangliosides into the microenvironment. The gangliosides act as modulators of the angiogenic response by promoting the angiogenic capacity of molecules, such as PGEl (Prostaglandin E1) or bFGF (Basic fibroblast growth factor), normally present in the tissue microenvironment. Their modulatory effect on angiogenesis may influence metastatic and/or primary tumor growth [79].

Vascular endothelial growth factor receptor-2 (VEGFR-2) signaling pathway is also observed in tumor angiogenesis. The studies suggest that GM3 inhibits VEGFR-2-mediated changes in vascular endothelial cell function and angiogenesis [80,81]. GM3 is an angiogenic inhibitor and perhaps could become a therapeutic avenue for tumor antiangiogenesis treatment. The various downstream signaling pathways or biological events can be regulated by GM3 through the inhibitory effect of VEGF-stimulated VEGFR-2 activation. GM3 suppressed VEGF induced VEGFR-2 activation by blocking its dimerization and also blocked the VEGF binding to VEGFR-2 through a GM3-specific interaction with the extracellular domain of VEGFR-2. GM3 is the only one which can directly interact with VEGFR-2, and inhibits VEGF/VEGFR-2-mediated biological function of vascular endothelial cell and angiogenesis.

GM3 can inhibit the VEGF-induced activation of PI-3K/AKT, extracellular signal-regulated kinases (ERK), vascular endothelial cadherin and focal adhesion kinase (FAK)/paxillin and suppresses proliferation, migration, and the tube formation caused by VEGF [80]. The exogenous GM3 inhibits VEGF-enhanced tyrosine phosphorylation of VEGFR-2, then the VEGF-induced downstream signal 
pathway of VEGFR-2 and cellular events are inhibited. In addition, the chick chorioallantoic membrane and Matrigel plug assays for neovascularization in response to VEGF with GM3 were also tested. GM3 strongly inhibited the formation of new blood vessels in the Matrigel plug with VEGF in C57BL/6 mice and GM3 significantly suppressed VEGF-induced neovascularization in the chick chorioallantoic membrane assay.

It is important to notice that one of the earliest steps leading to tumor angiogenesis is the enhanced vascular permeability. VEGF expressed in many tumors, and induces microvascular permeability, which can lead to tumor angiogenesis. Microvascular hyperpermeability by VEGF is involved in edema and the laying down of the fibrin-rich extracellular matrix through the extravasation of plasma and plasma proteins [80]. It has been confirmed that GM3 could lead to suppression of VEGF-stimulated microvessel permeability in mouse skin blood capillaries.

\section{EFFECT OF GANGLIOSIDE GM3 ON TUMOR MOTILITY}

Tumor cells adhesion, motility and migration are very important in the development and progression of cancers. Many studies have demonstrated that GM3 has a clear effect on cell adhesion, motility and migration, and change of GM3 expression plays multiple roles in the tumor cells motility, invasiveness and survival [21]. The adhesion of FUA169 cells (a mouse mammary carcinoma mutant cell line) to fibronectin needs the presence of GM3, and liposome adhesion to fibronectin-coated plates was significantly enhanced with GM3 at an optimal concentration. GM3 expression is not only related with greater cell-cell adhesion and slower growth presentation, but it also influences the cohesive properties of cells growth. In general, transformed or malignant cells, that contain GM3 as the major ganglioside, grow as clumps or islands in culture, but cells containing GM3 as a minor ganglioside grow as a fusiform monolayer [82].

GM3 has been identified as a cofactor of CD9, which is a motility-regulatory membrane receptor. In regulating tumor cell motility, CD9 complexed with GM3 inhibits motility. The enhanced synthesis of GM3 induced by brefeldin A caused decreased invasiveness was observed in some tumors including bladder cancer and colonic cancer due to the capability of GM3 to interconnect integrin with CD9 [6]. Moreover, it has been reported that GM2/GM3 complex fixed on silica nanospheres strongly inhibits cells motility through CD82/cMet-mediated pathway [83]. Some other reports also explain that HGFR/cMet is involved in the regulation of cell motility and migration, which is much more 
complicated [63]. Further studies showed that GM3 and GM2, independently or through the formation of GM3/GM2 dimers, can negatively regulate the motility through inhibiting c-Src (Proto-oncogene tyrosine-protein kinase) or cMet tyrosine kinases signaling pathways in the tetraspanin-expressing normal ant tumor cells [84]. The effect of GM3 synthase over expression on ovarian cancer cells motility is due to the ganglioside content variation. And the cell motility can also be reduced by the exogenous addition of GM3, GM2 and GM1 (not GD1a) as well as pharmacologically achieved higher ganglioside levels in A2780 cells [85,86].

\section{EFFECT OF GANGLIOSIDE GM3 ON MEMBRANE MICRODOMAINS}

The concept of membrane microdomains is that GSLs (GM3), GFRs, and other signaling molecules (TSs and integrins) are localized and functionalized as clusters in the membrane. Further studies indicated that membrane microdomains are involved in cell interaction, adhesion and related signal transduction. Horejsi's group reported an association of Src family kinases with glycosylphosphatidylinositol (GPI) anchored membrane proteins [87], and then detected GM3 in kinase / GPI-anchored protein complexes in human T-cell and myeloid cell lines [88]. Sonnino's group also demonstrated that GM3 synthase over expression in human ovarian cancer cells reduces cell motility by modulating caveolin expression and regulating c-Src kinase activity in the glycolipid-enriched microdomains $[85,86]$.

The melanoma B16 cells need GM3 to adhere to plates coated with a sialo-GM2 (Gg3) or LacCer on the cell surface based on carbohydrate - carbohydrate interaction [89-91]. More than $90 \%$ of total GM3 was found in the glycolipid-enriched microdomain fraction, along with several signal transducer molecules, such as c-Src, RhoA, H-Ras, FAK. When the fraction was immunoprecipitated with anti-GM3 mAb DH2, RhoA and c-Src were also co-precipitated with GM3 [92]. Tyrosine phosphorylation of FAK was strongly enhanced in B16 cells placed on Gg3-coated plates, and the phosphorylation was induced by GM3/Gg3 interaction. Along with FAK activation, activation of Ras and Rho were also enhanced [93].

Other studies of B16 cells revealed the presence of "GSL signaling domains", which is different from caveolin-containing membrane domains [93]. In mouse neuroblastoma Neuro2a cells, studies found that GM3 enriched microdomains were involved in signal transduction during neurite formation [94]. Moreover, Hakomori's group further indicates that clustered GSLs associated with c-Src, small 
G-protein (Rho A), and FAK are involved in GSL-dependent cell adhesion coupled with activation of signal transducers $[95,96]$. A typical example is GM3 ganglioside clustering at the cell surface of mouse melanoma B16, forming a "glycosignaling domain" (GSD) $[93,97]$. GM3 may have a role in initiating adhesion of melanoma to endothelial cells, the first step in metastasis [98]. Antigenicity, mediation of adhesion, and initiation of signaling through GM3 ganglioside at the B16 cell surface are thought to be maintained by GM3 clustering in GSD. If GM3 clustering in GSD is inhibited, antigenicity, adhesion, and signaling through GM3 could be blocked.

The "glycosynapse", named in similarity with "immunological synapse" [99,100], is highly enriched in TSs, hydrophobic proteolipids, integrins, and a wider range of signaling molecules and GFRs. Subsequent studies demonstrate that glycosynapses are involved in various cellular functions, such as cells growth and motility, which are enhanced during tumor progression [101]. Studies also showed that increased GM3 expression enhanced the anti-metastatic functions of TSs, CD82 and CD9 [102]. In a human colon cancer cell line HRT18 that highly expresses CD9, the endogenous or exogenous increase of cell surface GM3 expression resulted in increasing interaction of CD9 with integrin $\alpha 3$ and the decreasing cells migration through laminin-5-coated membranes [103]. In human diploid embryonic lung fibroblast WI38 cells, GM3 depletion by preincubation with the GlcCer synthase inhibitor P4 (DL-threo-1-phenyl-2-palmitoylamino-3-pyrrolidino-1-propanol) suppressed the contact inhibition of cells growth. Then at high cell density, FGF-induced cells growth was observed in P4-treated cells [4].

To regulate cell motility of GM3 referred to in Part 7, GM3-enriched microdomains also play an important role. Three kinds of bladder cancer cell lines have been studied [104], and show different cells motility and GM3 expression. The TS, integrin, and c-Src levels in glycosynaptic microdomains were analyzed. Through the study, the scheme of the inhibitory or suppressive effects for GM3 on cells motility and growth was established (Figure 7). First, GM3 enhances interaction of CD9 with integrin $\alpha 3 \beta 1$ and suppresses c-Src activation by the Ln-induced integrin activation. Then, GM2 alone and GM2/GM3 complex bind with CD82, but the former binding is weaker, and the complex can inhibit HGF-induced activation of c-Met and the cross-talk between c-Met and integrin $\alpha 3 \beta 1$. Further, various signal transducer molecules such as FAK, Src, Ras, Raf and MAPK (mitogen-activated protein kinase) are involved. Finally, the cell adhesion, motility or growth are controlled [83,105]. These results demonstrated that GM3 inhibits cells motility through modulating functions of other molecules 
co-localized in membrane microdomains.

\section{Figure 7}

\section{CONCLUSIONS}

GM3 is strongly related to cancers, and more and more evidences indicate that GM3 has an obvious effect on tumor development and progression. GM3 can modulate the cancer cell proliferation, adhesion, invasion and apoptosis. GM3 should become a promising target for cancer therapy. Several mechanisms that underline the actions of GM3 have been reported. GM3 interacts with basal membrane components, such as adhesion molecules, to regulate cell adhesion and migration, and GM3 directly interacts with GFRs, including EGFR, VEGER, to modulate the receptor function and subsequently affect the intracellular signaling pathways. Furthermore GM3 can modulate functions of other molecules co-localized in membrane microdomains. Thus, the mechanisms which involve GM3 function are particularly complicated, and the ability of GM3 to regulate cell behavior depends greatly on the tumor cell milieu and the expression levels of molecules associated to GM3.

\section{Acknowledgments:}

We thank the China Scholarship Council (CSC) for a Ph.D. fellowship to Changping ZHENG. Financial supports from the Centre National de la Recherche Scientifique (CNRS) and the Université Pierre et Marie Curie (UPMC) are gratefully acknowledged.

\section{References:}

[1] Hakomori, S. Traveling for the glycosphingolipid path. Glycoconj. J., 2000, 17, 627-647.

[2] Birklé, S.; Zeng, G.; Gao, L.; Yu, R.; Aubry, J. Role of tumor-associated gangliosides in cancer progression. Biochimie, 2003, $85(3-4), 455-463$.

[3] Kwak, D.H.; Ryu, J.S.; Kim, C.H.; Ko, K.; Ma, J.Y.; Hwang, K.A.; Choo, Y.K. Relationship between ganglioside expression and anti-cancer effects of the monoclonal antibody against epithelial cell adhesion molecule in colon cancer. Exp. Mol. Med., 2011, 43(12), 693-701.

[4] Toledo, M.S.; Suzuki, E.; Handa, K.; Hakomori, S. Cell growth regulation through GM3-enriched microdomain (glycosynapse) in human lung embryonal fibroblast WI38 and its oncogenic transformant VA13. J. Bio. Chem., 2004, 279(33), $34655-34664$.

[5] Chung, T.W.; Choi, H.J.; Kim, S.J.; Kwak, C.H.; Song, K.H.; Jin, U.H.; Chang, Y.C.; Chang, H.W.; Lee, Y.C.; Ha, K.T.; Kim, C.H. The Ganglioside GM3 is associated with cisplatin-induced apoptosis in human colon cancer cells. Plos One, 2014, 9 (5), e92786.

[6] Ono, M.; Sonnino, S.; Withers, D.A.; Nagai, H.; Hakomori, S. GM3 ganglioside inhibits CD9-facilitated haptotactic cell 
motility: coexpressionof GM3 and CD9 is essential in the downregulation of tumor cell motility and malignancy. Biochem., 2001, 40, 6414-6421.

[7] Qu, H.; Liu, J.; Wdzieczak-Bakala, J.; Lu, D.; He, X.; Sun, W.; Sollogoub, M.; Zhang, Y. Synthesis and cytotoxicity assay of four ganglioside GM3 analogues. Eur. J. Med. Chem., 2014, 75, 247-257.

[8] Bremer, E.G.; Schlessinger, J.; Hakomori, S. Ganglioside-mediatedmodulation of cell growth. Specific effects of GM3 on tyrosine phosphorylationof the epidermal growth factor receptor. J. Biol. Chem., 1986, 261, 2434-2440.

[9] Noll, E.N.E.; Lin, J.; Nakatsuji, Y.; Miller, R.H.; Black, P.M. GM3 as a novel growth regulator for human gliomas. Exp. Neurol., 2001, 168(2), 300-309.

[10] Manfredi, M.G.; Lim, S.; Claffey, K.P.; Seyfried, T.N. Gangliosides influence angiogenesis in an experimental mouse brain tumor. Cancer Res., 1999, 59, 5392-5397.

[11] Seyfried, T.N.; Mukherjee, P. Ganglioside GM3 is antiangiogenic in malignant brain cancer. J. Oncol., 2010, 2010, 961243.

[12] Ryuji,W.; Chikara, O.; Hiroshi, A.; Toshiko, T.; Makoto, S.; Seiichi, S.; Senji, H.; Atsushi, Ishii.; Masaki, S.; Yoichi, A. Ganglioside GM3 overexpression induces apoptosis and reduces malignant potential in murine bladder cancer. Cancer Res., 2002, 62, 3850-3854.

[13] Meuillet, E.J.; Kroes, R.; Yamamoto, H.; Warner, T.G.; Ferrari, J.; Mania-Farnell, B.; George, D.; Rebbaa, A.; Moskal, J.R.; Bremer, E.G. Sialidase gene transfection enhances epidermal growth factor receptor activity in an epidermoid carcinoma cell line, A431. Cancer Res., 1999, 59, 234-240.

[14] Kawamura, S.; Ohyama, C.; Watanabe, R.; Satoh, M.; Saito, S.; Hoshi, S.; Gasa, S.; Orikasa, S. Glycolipid composition in bladder tumor: a crucial role of GM3 ganglioside in tumor invasion. Int. J. Cancer, 2001, 94(3), 343-347.

[15] Gu, Y.; Zhang, J.; Mi, W.; Yang, J.; Han, F.; Lu, X.; Yu, W. Silencing of GM3 synthase suppresses lung metastasis of murine breast cancer cells. Breast Cancer Res., 2008, 10(1), 1-12.

[16] Chefalo, P.; Pan, Y.; Nagy, N.; Guo, Z.; Harding, C. Efficient metabolic engineering of GM3 on tumor cells by $N$-Phenylacetyl-D-mannosamine. Biochem., 2006, 45, 3733-3739.

[17] Hasegawa, T.; Yamaguchi, K.; Wada, T.; Takeda, A.; Itoyama, Y.; Miyagi, T. Molecular cloning of mouse ganglioside sialidase and its increased expression in neuro2a cell differentiation. J. Bio. Chem., 2000, 275, 8007-8015.

[18] Papini, N.; Anastasia, L.; Tringali, C.; Croci, G.; Bresciani, R.; Yamaguchi, K.; Miyagi, T.; Preti, A.; Prinetti, A.; Prioni, S.; Sonnino, S.; Tettamanti, G.; Venerando, B.; Monti, E. The plasma membrane-associated sialidase MmNEU3 modifies the ganglioside pattern of adjacent cells supporting its involvement in cell-to-cell interactions. J. Bio. Chem., 2004, 279(17), 16989-16995.

[19] Hakomori, S.; Handa, K. GM3 and cancer. Glycoconj. J., 2015, 32(1-2), 1-8.

[20] Tringali, C.; Lupo, B.; Silvestri, I.; Papini, N.; Anastasia, L.; Tettamanti, G.; Venerando, B. The plasma membrane sialidase NEU3 regulates the malignancy of renal carcinoma cells by controlling beta1 integrin internalization and recycling. $J$. Bio. Chem., 2012, 287(51), 42835-42845.

[21] Ueno, S.; Saito, S.; Wada, T.; Yamaguchi, K.; Satoh, M.; Arai, Y.; Miyagi, T. Plasma membrane-associated sialidase is up-regulated in renal cell carcinoma and promotes interleukin-6-induced apoptosis suppression and cell motility. J. Bio. Chem., 2006, 281(12), 7756-7764.

[22] Masashi, S.; Seiichi, S.; Ryuzaburo, S.; Susumu, S.; Takao, Y.; Takashi, T.; Reiji, K.; Taeko, M. Reduced sialidase expression in highly metastatic variants of mouse colon adenocarcinoma 26 and retardation of their metastatic ability by sialidase overexpression. Int. J. Cancer, 2002, 97, 180-185.

[23] Hersey, P.; Jamal, O.; Henderson, C.; Zardawi, I.; D'Alessandro, G. Expression of the gangliosides GM3, GD3 and GD2 in tissue sections of normal skin, naevi, primary and metastatic melanoma. Int. J. Cancer, 1988, 41, 336-343.

[24] Ichikawa, S.; Nakajo, N.; Sakiyama, H.; Hirabayashi, Y. A mouse B16 melanoma mutant deficient in glycolipids. Biochem., 1994, 91, 2703-2707. 
[25] Inokuchi, J.; Jimbo, M.; Kumamoto, Y.; Shimeno, H.; Nagamatsu, A. Expression of ganglioside GM3 and H-2 antigensin clones with different metastatic and growthpotentials isolated from Lewis lung carcinoma(3LL) cell line. Clin. Exp. Metastasis, 1993, $11,27-36$.

[26] Tringali1, C.; Silvestri1, I.; Testa, F.; Baldassari, P.; Anastasia, L.; Mortarini, R.; Anichini, A.;López-Requena, A.; Tettamanti, G.; Venerando, B. Molecular subtyping of metastatic melanoma based on cell ganglioside metabolism profiles. BMC Cancer, 2014, 14, 560 .

[27] Rosaria Bassi, P.V.; Paola, G.; Laura, R.; Guido, T. GM3 ganglioside inhibits endothelin-1-mediated signal transduction in C6 glioma cells. FEBS Lett., 2001, 507, 101-104.

[28] Samraj, A.N.; Laubli, H.; Varki, N.; Varki, A. Involvement of a non-human sialic acid in human cancer. Front. Oncol., 2014, 4,33.

[29] van Cruijsen, H.; Ruiz, M.G.; van der Valk, P.; de Gruijl, T.D.; Giaccone, G. Tissue micro array analysis of ganglioside $\mathrm{N}$-glycolyl GM3 expression and signal transducer and activator of transcription (STAT)-3 activation in relation to dendritic cell infiltration and microvessel density in non-small cell lung cancer. BMC Cancer, 2009, 9, 180.

[30] Blanco, R. N-Glycolyl GM3 ganglioside as a relevant tumor antigen in humans. J. Mol. Bio. Diagnosis, 2016, 07(06), $1000 \mathrm{e} 124$.

[31] Casadesus, A.V.; Fernandez-Marrero, Y.; Clavell, M.; Gomez, J.A.; Hernandez, T.; Moreno, E.; Lopez-Requena, A. A shift from N-glycolyl- to N-acetyl-sialic acid in the GM3 ganglioside impairs tumor development in mouse lymphocytic leukemia cells. Glycoconj. J., 2013, 30(7), 687-699.

[32] Pochechueva, T.; Jacob, F.; Fedier, A.; Heinzelmann-Schwarz, V. Tumor-associated glycans and their role in gynecological cancers: accelerating translational research by novel high-throughput approaches. Metabolites, 2012, 2(4), 913-939.

[33] Dorvignit, D.; Garcia-Martinez, L.; Rossin, A.; Sosa, K.; Viera, J.; Hernandez, T.; Mateo, C.; Hueber, A.O.; Mesa, C.; Lopez-Requena, A. Antitumor and cytotoxic properties of a humanized antibody specific for the GM3(Neu5Gc) ganglioside. Immunobiology, 2015, 220(12), 1343-1350.

[34] Krengel, U.; Bousquet, P.A. Molecular recognition of gangliosides and their potential for cancer immunotherapies. Front. Immuno, 2014, 5, 325.

[35] Gridelli, C.; Rossi, A.; Maione, P.; Ferrara, M.L.; Castaldo, V.; Sacco, P.C. Vaccines for the treatment of non-small cell lung cancer: a renewed anticancer strategy. Oncologist, 2009, 14(9), 909-920.

[36] Osorio, M.; Gracia, E.; Rodríguez, E.; Saurez, G.; del Carmen Arango, M.; Noris, E.; Torriella, A.; Joan, A.; Gómez, E.; Anasagasti, L.; González, J.L.; de los Angeles Melgares, M.; Torres, I.; González, J.; Alonso, D.; Rengifo, E.; Carr, A.; Pérez, R.; Pérez, R.; Enrique Fernández, L. Heterophilic NeuGcGM3 ganglioside cancer vaccine in advanced melanoma patients: Results of a phase Ib/IIa study. Cancer Biol. Ther, 2014, 7(4), 488-495.

[37] Gabri, M.R.; Ripoll, G.V.; Alonso, D.F.; Gomez, D.E. Role of cell surface GM3 ganglioside and sialic acid in the antitumor activity of a GM3-based vaccine in the murine B16 melanoma model. Cancer Res. Clin. Oncol, 2002, 128(12), 669-677.

[38] Mazorra, Z.; Mesa, C.; Fernández, L.E. GM3 ganglioside: a novel target for the therapyagainst melanoma. Biotecnol. Apl., 2009, 26, 256-259.

[39] Zheng, X.; Yang, F.; Zheng, M.; Huo, C.; Zhang, Y.; Ye, X. Improvement of the immune efficacy of carbohydrate vaccines by chemical modification on the GM3 antigen. Org. Biomol. Chem., 2015, 13(22), 6399-6406.

[40] Wang, Q.; Zhang, J.; Guo, Z. Efficient glycoengineering of GM3 on melanoma cell and monoclonal antibody-mediated selective killing of the glycoengineered cancer cell. Bioorg. Med. Chem., 2007, 15(24), 7561-7567.

[41] Miranda, A.; de Leon, J.; Roque-Navarro, L.; Fernandez, L.E. Cytofluorimetric evaluation of N-glycolylated GM3 ganglioside expression on murine leukocytes. Immunol. Lett., 2011, 137(1-2), 38-45.

[42] Oliva, J.P.; Valdes, Z.; Casaco, A.; Pimentel, G.; Gonzalez, J.; Alvarez, I.; Osorio, M.; Velazco, M.; Figueroa, M.; Ortiz, R.; Escobar, X.; Orozco, M.; Cruz, J.; Franco, S.; Diaz, M.; Roque, L.; Carr, A.; Vazquez, A.M.; Mateos, C.; Rubio, M.C.; Perez, R.; 
Fernandez, L.E. Clinical evidences of GM3 (NeuGc) ganglioside expression in human breast cancer using the 14F7 monoclonal antibody labelled with (99m)Tc. Breast Cancer Res. Tr., 2006, 96(2), 115-121.

[43] Fernandez, L.E.; Gabri, M.R.; Guthmann, M.D.; Gomez, R.E.; Gold, S.; Fainboim, L.; Gomez, D.E.; Alonso, D.F. NGcGM3 ganglioside: a privileged target for cancer vaccines. Clin. Dev. Immunol., 2010, 2010, 814397.

[44] de la Torre, A.; Hernandez, J.; Ortiz, R.; Cepeda, M.; Perez, K.; Car, A.; Viada, C.; Toledo, D.; Guerra, P.P.; Garcia, E.; Arbolaez, M.; Fernandez, L.E. NGlycolylGM3/VSSP Vaccine in metastatic breast cancer patients: Results of phase I/IIa clinical trial. Breast Cancer (Auckl), 2012, 6, 151-157.

[45] Segatori, V.I.; Otero, L.L.; Fernandez, L.E.; Gomez, D.E.; Alonso, D.F.; Gabri, M.R. Antitumor protection by NGcGM3/VSSP vaccine against transfected B16 mouse melanoma cells overexpressing N-Glycolylated gangliosides. In Vivo, 2012, 26, 609-618.

[46] Perez, K.; Osorio, M.; Hernandez, J.; Carr, A.; Fernandez, L.E. NGcGM3/VSSP vaccine as treatment for melanoma patients. Hum. Vacc. Immunother, 2013, 9(6), 1237-1240.

[47] de la Torre, A.; Perez, K.; Vega, A.M.; Santiesteban, E.; Ruiz, R.; Hernandez, L.; Durruti, D.; Viada, C.E.; Sanchez, L.; Alvarez, M.; Duran, Y.; Moreno, Y.G.; Arencibia, M.; Cepeda, M.; Domecq, M.; Cabrera, L.; Sanchez, J.L.; Hernandez, J.J.; Valls, A.R.; Fernandez, L.E. Superior efficacy and safety of a nonemulsive variant of the NGcGM3/VSSP vaccine in advanced breast cancer patients. Breast Cancer (Auckl), 2016, 10, 5-11.

[48] Voldborg, B.R.; Damstrup, L.; Spang-Thomsen, M.; Poulsen, H.S. Epidermal growth factor receptor (EGFR) and EGFR mutations, functionand possible role in clinical trials. Ann. Oncol., 1997, 8, 1197-1206.

[49] Bremer, E.G.; Hakomori, S.; Bowen-Pope, D.F.; Raines, E.; Ross, R. Ganglioside-mediated modulation of cell growth, growth factor binding, and receptor phosphorylation. J. Biol. Chem., 1984, 259, 6818-6825.

[50] Abdelhadi, R.; Jay H.; Hirotaka, Y.; Donna S.K.; Eric, G.B. Ganglioside GM3 inhibition of EGF receptor mediated signal transduction. Glycobiology, 1996, 6, 399-406.

[51] Yoon, S.J.; Nakayama, K.i.; Hikita, T.; Handa, K.; Hakomori, S. Epidermal growth factor receptor tyrosine kinase is modulated by GM3 interaction with N-linked GlcNAc termini of the receptor. Proc. Natl. Acad. Sci. USA, 2006, 103(50), 18987-18991.

[52] Wang, X.; Sun, P.; O'Gorman, M.; Tai, T.; Paller, A.S. Epidermal growth factor receptor glycosylation is required for ganglioside GM3 binding and GM3-mediated suppresion of activation. Glycobiology, 2001, 11, 515-522.

[53] Wang, X.Q.; Sun, P.; Paller, A.S. Ganglioside GM3 blocks the activation of epidermal growth factor receptor induced by integrin at specific tyrosine sites. J. Biol. Chem., 2003, 278(49), 48770-48778.

[54] Kovacs, E.; Das, R.; Wang, Q.; Collier, T.S.; Cantor, A.; Huang, Y.; Wong, K.; Mirza, A.; Barros, T.; Grob, P.; Jura, N.; Bose, R.; Kuriyan, J. Analysis of the role of the C-terminal tail in the regulation of the epidermal growth factor receptor. Mol. Cell. Biol., 2015, 35(17), 3083-3102.

[55] Pourazar, J.; Blomberg, A.; Kelly, F.J.; Davies, D.E.; Wilson, S.J.; Holgate, S.T.; Sandstrom, T. Diesel exhaust increases EGFR and phosphorylated C-terminal Tyr 1173 in the bronchial epithelium. Part. Fibre Toxicol., 2008, 5, 8.

[56] Shiozaki, K.; Yamaguchi, K.; Sato, I.; Miyagi, T. Plasma membrane-associated sialidase (NEU3) promotes formation of colonic aberrant crypt foci in azoxymethane-treated transgenic mice. Cancer Sci., 2009, 100(4), 588-594.

[57] Miyata, M.; Kambe, M.; Tajima, O.; Moriya, S.; Sawaki, H.; Hotta, H.; Kondo, Y.; Narimatsu, H.; Miyagi, T.; Furukawa, K.; Furukawa, K. Membrane sialidase NEU3 is highly expressed in human melanoma cells promoting cell growth with minimal changes in the composition of gangliosides. Cancer Sci., 2011, 102(12), 2139-2149.

[58] Miljan, E.A.; Meuillet, E.J.; Mania-Farnell, B.; George, D.; Yamamoto, H.; Simon, H.G.; Bremer, E.G. Interaction of the extracellular domain of the epidermal growth factor receptor with gangliosides. J. Biol. Chem., 2002, 277(12), 10108-10113.

[59] Zhou, Q.; Hakomori, S.; Kitamuras, K.; Igarashi, Y. GM3 Directly inhibits tyrosine phosphorylation and de-N-acety1-GM3 directly enhances serine phosphorylation of epidermal growth factor receptor, independently of receptor-receptor interaction. J. Biol. Chem., 1994, 269, 1950-1965. 
[60] Kawashima, N.; Qu, H.; Lobaton, M.; Zhu, Z.; Sollogoub, M.; Cavenee, W.K.; Handa, K.; Hakomori, S.; Zhang, Y. Efficient synthesis of chloro-derivatives of sialosyllactosylceramide, and their enhanced inhibitory effect on epidermal growth factor receptor activation. Oncol. Lett., 2014, 7(4), 933-940.

[61] Haga, Y.; Hatanaka, K.; Hakomori, S. Effect of lipid mimetics of GM3 and lyso-GM3 dimer on EGF receptor tyrosine kinase and EGF-induced signal transduction. Biochim. Biophys. Acta, 2008, 1780(3), 393-404.

[62] Huang, X.; Zhang, Y.; Xu, J.; Tian, Y.; Ma, K. Ganglioside GM3 inhibits hepatoma cell motility via down-regulating activity of EGFR and PI3K/AKT signaling pathway. J. Cell. Biochem. ,2013, 114(7), 1616-1624.

[63] Li, Y.; Huang, X.; Zhong, W.; Zhang, J.; Ma, K. Ganglioside GM3 promotes HGF-stimulated motility of murine hepatoma cell through enhanced phosphorylation of cMet at specific tyrosine sites and PI3K/Akt-mediated migration signaling. Mol. Cell. Biochem, 2013, 382(1-2), 83-92.

[64] Li, Y.; Huang, X.; Wang, C.; Li, Y.; Luan, M.; Ma, K. Ganglioside GM3 exerts opposite effects on motility via epidermal growth factor receptor and hepatocyte growth factor receptor-mediated migration signaling. Mol. Med. Rep., 2015, 11(4), 2959-2966

[65] Palomo, A.G.; Santana, R.B.; Pérez, X.E.; Santana, D.B.; Gabri, M.R.; Monzon, K.L.; Pérez, A.C. Frequent co-expression of EGFR and NeuGcGM3 ganglioside in cancer: it's potential therapeutic implications. Clin. Exp. Metas., 2016, 33(7), 717-725.

[66] Gomez-Mouton, C.; Abad, J.L.; Mira, E.; Lacalle, R.A.; Gallardo, E.; Jimenez-Baranda, S.; Illa, I.; Bernad, A.; Manes, S.; Martinez, A.C. Segregation of leading-edge and uropod components into specific lipid rafts during T cell polarization. Proc. Natl. Acad. Sci. USA, 2001, 98(17), 9642-9647.

[67] Wang, X.; Sun, P.; Go, L.; Koti, V.; Fliman, M.; Paller, A.S. Ganglioside GM3 promotes carcinoma cell proliferation via urokinase plasminogen activator-induced extracellular signal-regulated kinase-independent p70S6 kinase signaling. J. Invest. Dermatol., 2006, 126(12), 2687-2696.

[68] Wang, X.; Sun, P.; Paller, A.S. Gangliosides inhibit urokinase-type plasminogen activator (uPA)-dependent squamous carcinoma cell migration by preventing uPA receptor/alphabeta integrin/epidermal growth factor receptor interactions. J. Invest. Dermatol., 2005, 124(4), 839-848.

[69] Dufner, A.;Thomas, G. Ribosomal S6 kinase signaling and the control of translation. Exp. Cell Res., 1999, 253, 100-109.

[70] Abate, L.E.; Mukherjee, P.; Seyfried, T.N. Gene-linked shift in ganglioside distribution influences growth and vascularity in a mouse astrocytoma. J. Neurochem., 2006, 98(6), 1973-1984.

[71] Giulio, A.; Stefania, F.; Paola, S.; Francoise, M.; Pietro,P.;Federico, S.; Pier Mario, C.; Pietro, M.G. Influence of gangliosides on primary and metastatic neoplastic growth in human and murine cells. Cancer Res., 1987, 47, 4243-4247.

[72] Ravindranath, M.H.; Tsuchida, T.; Morton, D.L.; Irie, R.F. Ganglioside GM3:GD3 ratio as an indexfor the management of melanoma.Cancer, 1991, 67, 3029-3035.

[73] Seyfried, T.N.; el-Abbadi, M.; Roy, M.L. Ganglioside distributionin murine neural tumors. Mol. Chem. Neuropathol., 1992, $17,147-167$.

[74] Ecsedy, J.A.; Holthaus, K.A.; Yohe, H.C.; Seyfried, T.N. Expression of mouse sialic acid on gangliosides of a human glioma grown as a xenograft in SCID mice. $J$.Neurochem., 1999, 73, 254-259.

[75] Margheri, F.; Chillà, A.; Laurenzana, A.; Serratì, S.; Mazzanti, B.; Saccardi, R.; Santosuosso, M.; Danza, G.; Sturli, N., Rosati, F., Magnelli, L.; Papucci, L.; Calorini, L.; Bianchini, F.; Del Rosso, M.; Fibbi, G. Endothelial progenitor cell-dependent angiogenesis requires localization of the full-length form of uPAR in caveolae. Blood, 2011, 118, 3743-55.

[76] Chillà, A.; Magherini, F.; Margheri, F.; Laurenzana, A.; Gamberi, T.; Bini, L.; Bianchi, L.; Danza, G.; Mazzanti, B.; Serratì, S.; Modesti, A.; Del Rosso, M.; Fibbi, G. Proteomic Identification of VEGF-dependent protein enrichment to membrane caveolar-raft microdomains in endothelial progenitor cells. Mol. Cell Proteomics, 2013, 12, 1926-1938.

[77] Margheri, F.; Papucci, L.; Schiavone, N.; D’Agostino, R.; Trigari, S.; Serrat, S.; Laurenzana, A.; Biagioni, A.; Luciani, C.; Chill, A.; Andreucci, E.; Del Rosso, T.; Margheri, G.; Del Rosso, M.; Fibbi, G. Differential uPAR recruitment in caveolar-lipid rafts by GM1 and GM3 gangliosides regulates endothelial progenitor cells angiogenesis. J. Cell Mol. Med., 2015, 19, 113-123. 
[78] Alessandri, G; Cornaglia-Ferraris, P.; Gullino, P.M. Angiogenic and angiostatic microenvironment in tumors: role of ganglioside. Acta Oncol., 1997, 36, 383-387.

[79] Gullino, P.M. Prostaglandins and gangliosides of tumor microenviroment: their role in angiogenesis. Acta Oncol., 1995, $34,439-441$.

[80] Chung, T.W.; Kim, S.J.; Choi, H.J.; Kim, K.J.; Kim, M.J.; Kim, S.H.; Lee, H.J.; Ko, J.H.; Lee, Y.C.; Suzuki, A.; Kim, C.H. Ganglioside GM3 inhibits VEGF/VEGFR-2-mediated angiogenesis: direct interaction of GM3 with VEGFR-2. Glycobiology, 2009, 19(3), 229-239.

[81] Mukherjee, P.; Faber, A.C.; Shelton, L.M.; Baek, R.C.; Chiles, T.C.; Seyfried, T.N. Thematic review series: sphingolipids.Ganglioside GM3 suppresses the proangiogenic effects of vascular endothelial growth factor and ganglioside GD1a. J. Lipid Res., 2008, 49(5), 929-938.

[82] Bai, H.; Seyfried, T.N. Influence of ganglioside GM3 and high density lipoprotein on the cohesion of mouse brain tumor cells. J. Lipid Res., 1997, 38, 160-172.

[83] Todeschini, A.R.; Dos Santos, J.N.; Handa, K.; Hakomori, S. Ganglioside GM2/GM3 complex affixed on silica nanospheres strongly inhibits cell motility through CD82/cMet-mediated pathway. Proc. Natl. Acad. Sci. USA, 2008, 105(6), 1925-1930.

[84] Mitsuzuka, K.; Handa, K.; Satoh, M.; Arai, Y.; Hakomori, S. A specific microdomain ("glycosynapse 3") controls phenotypic conversion and reversion of bladder cancer cells through GM3-mediated interaction of alpha3beta1 integrin with CD9. J. Bio. Chem., 2005, 280(42), 35545-35553.

[85] Prinetti, A.; Cao, T.; Illuzzi, G.; Prioni, S.; Aureli, M.; Gagliano, N.; Tredici, G.; Rodriguez-Menendez, V.; Chigorno, V.; Sonnino, S. A glycosphingolipid/caveolin-1 signaling complex inhibits motility of human ovarian carcinoma cells. J. Biol. Chem., 2011, 286(47), 40900-40910.

[86] Prinetti, A.; Aureli, M.; Illuzzi, G.; Prioni, S.; Nocco, V.; Scandroglio, F.; Gagliano, N.; Tredici, G.; Rodriguez-Menendez, V.; Chigorno, V.; Sonnino, S. GM3 synthase overexpression results in reduced cell motility and in caveolin-1 upregulation in human ovarian carcinoma cells. Glycobiology, 2010, 20(1), 62-77.

[87] Stefanová, I.; Horeisi, V.; Ansotegui, I.J.; Knapp, W.; Stockinger, H. GPI-anchored cell-surface molecules complexed to protein tyrosine kinases. Sci., 1991, 254, 1016-1019.

[88] Kniep, B.; Cinek, T.; Angelisova, P.; Horejsi, V. Association of theGPI-anchored leucocyte surface glycoproteins with ganglioside GM3. Biochem. Biophys. Res. Commun., 1994, 203, 1069-1075.

[89] Handa, K.; Hakomori, S. Carbohydrate to carbohydrate interaction in development process and cancer progression. Glycoconj. J., 2012, 29(8-9), 627-637.

[90] Kojima, N.; Hakomori, S. Specific interaction betweengangliotriaosylceramide (Gg3) and sialosyllactosylceramide (GM3)as a basis for specific cellular recognition between lymphoma andmelanoma cells. J. Biol. Chem., 1989, 264, 20159-20162.

[91] Kojima, N.; Hakomori, S. Cell adhesion, spreading, and motility of GM3-expressing cells based glycolipid-glycolipid interaction. J. Biol. Chem., 1991, 266, 17552-17558.

[92] Yamamura, S.; Handa, K.; Hakomori, S. A close association of GM3with c-Src and Rho in GM3-enriched microdomains at the B16 melanomacell surface membrane: a preliminary note. Biochem. Biophys. Res. Commun., 1997, 236, $218-222$.

[93] Iwabuchi, K.; Handa, K.; Hakomori, S. Separation of "glycosphingolipid signaling domain" from caveolin-containingmembrane fraction in mouse melanoma B16 cells and its role in celladhesion coupled with signaling. J. Biol. Chem., 1998, 273, 33766-33773.

[94] Prinetti, A.; Iwabuchi, K.; Hakomori, S. Glycosphingolipid-enrichedsignaling domain in mouse neuroblastoma neuro2a cells.Mechanism of ganglioside-dependent neuritogenesis. J. Biol. Chem., 1999, 274, 20916-20924.

[95] Iwabuchi, K.; Yamamura, S.; Prinetti, A.; Handa, K.; Hakomori, S. GM3-enriched microdomain involved in cell adhesion and signal transduction through carbohydrate-carbohydrate interaction in mouse melanoma B16 cells. J. Biol. 
Chem., 1998, 273, 9130-9138.

[96] Iwabuchi, K.; Zhang, Y.; Handa, K.;Withers, D.A.; Sinay, P.; Hakomori, S. Reconstitution of membranes simulating “Glycosignaling Domain”and their susceptibility to Lyso-GM3. J. Biol. Chem., 2000, 275, 15174-15181.

[97] Zhang, Y.; Iwabuchi, K.; Nunomura, S.;Hakomori, S. Effect of synthetic sialyl 2-1 sphingosine and other glycosylsphingosines on the structure and function of the "Glycosphingolipid Signaling Domain (GSD)" in mouse melanoma B16 cells. Biochem., 2000, 39, 2459-2468.

[98] Kojima, N.; Shiota, M.; Sadahira, Y.; Handa, K.; Hakomori, S. Cell adhesion in a dynamic flow system as comparedto static system. J. Biol. Chem., 1992, 267, 17264-17270.

[99] Bromley, S.K.; Burack, W.R.; Johnson, K.G.; Somersalo, K.; Sims,T.N.; Sumen, C.; Davis, M.M.; Shaw, A.S.; Allen, P.M.; Dustin,; M.L. The immunological synapse. Ann. Rev. Immun., 2001, 19, 375-396.

[100] Viola, A.; Schroeder, S.; Sakakibara, Y.; Lanzavecchia, A. T lymphocytecostimulation mediated by reorganization of membrane microdomains. Sci., 1999, 283, 680-682.

[101] Hakomori, S. Glycosylation defining cancer malignancy: new wine in an old bottle. Proc. Natl. Acad. Sci. USA, 2002, 99(16), 10231-10233.

[102] Ono, M.; Handa, K.; Withers, D.A.; Hakomori, S. Motility inhibitionand apoptosis are induced by metastasis-suppressinggene product CD82 and its analogue CD9, with concurrent glycosylation. Cancer Res., 1999, 59, 2335-2339.

[103] Kawakami, Y.; Kawakami, K.; Steelant, W.F.; Ono, M.; Baek, R.C.; Handa, K.; Withers, D.A.; Hakomori, S. Tetraspanin CD9 is a "proteolipid," and its interaction with alpha 3 integrin in microdomain is promoted by GM3 ganglioside, leading to inhibition of laminin-5-dependent cell motility. J. Biol. Chem., 2002, 277(37), 34349-34358.

[104] Wang, H.; Isaji, T.; Satoh, M.; Li, D.; Arai, Y.; Gu, J. Antitumor effects of exogenous ganglioside GM3 on bladder cancer in an orthotopic cancer model. Urology, 2013, 81(1), 210 e11-e15.

[105] Todeschini, A.R.; Dos Santos, J.N.; Handa, K.; Hakomori, S. Ganglioside GM2-tetraspanin CD82 complex inhibits met and its cross-talk with integrins, providing a basis for control of cellmotility through glycosynapse. J. Biol. Chem., 2007, 282(11), $8123-8133$. 


\section{Figure legends}

Figure 1. Structure of ganglioside GM3. It contains sialic acid, lactose and ceramide.

Figure 2. Structure of two ganglioside GM3 analogues.

Figure 3. The epidermal growth factor receptor (EGFR) activation by EGF. The EGF can bind to EGFR resulting in dimerisation, autophosphorylation, and activating downstream signaling.

Figure 4. Structure of lipid mimetic of lyso-GM3 dimer.

Figure 5. Mechanism of GM3-triggered uPAR activation and cell proliferation. uPA can be able to stimulate cell proliferation by either ERK-dependent or ERK-independent pathways, and the mechanism is different. In contrast to (a) control cells, cells that (b) over express GM3 show activation of PI-3K and protein kinase C PKC $\zeta$, increased p70S6 kinase activity, and cell proliferation, despite suppression of ERK signaling. Cells in which GM3 is (c) depleted show activation of ERK signaling, leading to increased cell proliferation that is independent of p70S6 kinase activation.

Figure 6. GM3 reduces CT-2A tumor vascularity when added to the tumor microenvironment. Small fragments of the CT-2A tumor were grown in Matrigel that contained either no GM3 (control) or GM3 $(40 \mu \mathrm{M})$. The tumor was grown in Matrigel for approximately two weeks in the flank of the syngeneic host C57BL/6 mice.

Figure. 7 Scheme of the inhibitory or suppressive effects for GM3 on cell motility and growth through modulating functions of other molecules co-localized in membrane microdomains. First, GM3 enhances interaction between CD9 and integrin $\alpha 3 \beta 1$ and suppresses c-Src activation through the Ln-induced integrin activation. Further, GM2 alone and GM2/GM3 complex bind with CD82, then can inhibit HGF-induced activation of c-Met and the cross-talk between c-Met and integrin $\alpha 3 \beta 1$. Finally, various signal transducer molecules such as FAK, Src, Ras, Raf and MAPK are involved, resulting in the control of cell adhesion, motility or growth. 
